\title{
Ueber Erdströme während des Nordlichts 1894 Febr. 22.
}

\author{
(Auszug aus einem Schreiben an den Herausgeber.)
}

There was a remarkable localization both of the / comparison. That there is a localization of auroras and aurora and of earth currents affecting the telegraph lines, earth currents at certain meridians at times is most interover the western half of the North American Continent on esting. The Western Union Telegraph which extends widely Febr. $22^{\text {nd }} 1894$, and a somewhat similar but not so over North America is supplying memoranda of the geostrongly marked localization over the eastern part of the graphical distribution of earth currents on their lines for continent on the day following. It would be of interest/the purpose of comparison with the similar record of to have as full notes as possible especially in regard to auroras, and any such records from other sources will be earth currents in other parts of the world for purposes of ! of service.

Lyons, N. Y., 1894 April. 30.

M. Aeeder.

\section{Todes-Anzeige.}

Am 17. Mai d. J. starb Professor Dr. Fischer, Sectionschef im Königlich Preussischen Geodätischen Institut und Centralbureau der Internationalen Frdnessung, in Folge eines langjährigen Herzleidens.

Amand Fischer wurde an 10. December $183^{6}$ zu Deutsch-Leippe bei Grottkau in Schlesien als Sohn eines Schullehrers geboren, besuchte das Mathias-Gymnasium in Breslau und studirte daselbst, Anfangs fur den geistlichen Beruf bestimmt, Mathematik und Naturwissenschaften. Nachdem er einige Jahre Hauslehrer gewesen war, promovirte er I 866 auf Grund einer Dissertation über den Cometen I860 III.

Mit seinem Eintritt in das Centralbureau der Mitteleuropäischen Gradmessung im Jahre 1867 und in das 1869 neu errichtete, damit vereinigte Königl. Preussische Geodätische Institut beginnt die Thätigkeit, der er seine Kräfte bis zuletzt gewidmet hat. Vom 1. Juli 1877 an leitete er eine der geodaitischen Sectionen.

Unter den von ihm veröfentlichten Arbeiten ist zuvörderst das w Rheinische Dreiecksnetz* (Netzausgleichung, 1882) hervorzuheben, an dessen umfangreichen Messungen Fischer auf den meisten Stationen selbst betheiligt war. Er fand dabei Anlass, sich mit der Lateralrefraction zu beschäftigen. Die, Lothabweichungen in der Umgebung von Berlin (1889) lieferten einen interessanten Beitrag fur die neuerdings in den Vordergrund getretenen Beziehungen der Geodäsie zur Geologie. Früher hatte er in einem Artikel der Astronomischen Nachrichten (13d. 88) die Gestalt der Erde und dic Pendelmessungen die Abweichung der von Ph. Fischer aus den Schweremessungen gefolgerten Abplattung von der geodätisch berechneten auf geologische Ursachen zurückgefuhrt, wobei er den heutigen Anschauungen nahe kam. Die Veröffentlichung des, Berliner Basisnetzess ( 1891 ) knüpft an eine Thätigkeit an, die ihn besonders in Anspruch nahm. An den Messungen der Strehlener, Berliner und Bonner Basis hat er, zuletzt als Leiter dieser Operationen, einen hervorragenden Antheil genommen. Thernoelektrische Untersuchungen über die Ausdehnung der Basisstangen sind ihm zu verdanken (Astr. Nachr. Hd. 103). Die Berechnung der von ihm ausgefuhrten trigonometrischen Nivellements zwischen mehreren Nordsee-Inseln und den Festlande vollendet zu sehen, war ihm nicht mehr vergönnt. Bei Gelegenheit dicser Messungen bestimmte er den Längenunterschied zwischen Wangeroog und Schillig durch optische Signale (Astr. Nachr. 13d. I 24 ).

Die literarische Thätigkeit Fischer's kennzeichnct aber nur zum 'Theil seine Wirksamkeit, man musste ihn als Heobachter sehen, um seine Gewandtheit und Sorgfalt, vor Allem aber seine liebe zur Sache und Aufopferungsfähigkeit kennen zu lernen. Neben den geodatischen Messungen hat er an zahlreichen Stationen astronomische Beobachtungen für die Zwecke der Erdmessung angestellt.

Aus einem glïcklichen Fanilienleben ist er nach geduldig getragenen Leiden abgerufen worden. Mit seiner Wittwe und drei Kindern trauern um ihn seine Collegen, bei denen er, auch als Mensch, durch seine herzliche Freundlichkeit und seine lautere Gesinnung sich ein bleibendes Gedächtniss gesichert hat.

\section{In halt:}

Zu N'r. 3235. E. Weiss. Beubachtungen auf der k. k. Sternwarte in Wien. 337. - L. Lamp. Peohachtungen des Cometen 1894 I (Denning Mä\% 26). 343. - H. C. Russell. Obscrvations of Comet $1894 \ldots$ (Ciale April 1). 343 - - R. L. Y. Ellery. Elements and Ephemeris of Comet 1894...(Gale April 1). 345. - 7. G. Galle. Oppositions-Ephemeride des Planeten (84) KLio. 345. - E. Millosevish Elementi ellittici di (303) Josephina e (306) Unitas. 347. - A. Berberich. Elemente von kieinen Planeten. 347. - A. Berberich. Elemente und Ephemeride des Planeten (324). 347. - A. Berberich. Ephemeride des Pianeten (i75) Andromache. 349. H. Kobold. Berichtigung. 349. - M. A. Veeder. Leber Erdstrüme während des Nordlichts I 894 Febr. 22. 351. - Todes-Anzeige. 351.

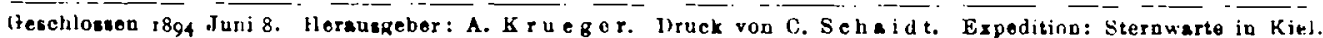

